\title{
ARTICLE OPEN \\ Characterization of domain distributions by second harmonic generation in ferroelectrics
}

Yuan Zhang ${ }^{1}$, Yi Zhang $\mathbb{D}^{1}$, Quan Guo ${ }^{2}$, Xiangli Zhong ${ }^{1}$, Yinghao Chu iD $^{3}$, Haidong Lu ${ }^{4}$, Gaokuo Zhong (iD ${ }^{1}$, Jie Jiang ${ }^{1}$, Congbing Tan $^{1}$, Min Liao', Zhihui Lu ${ }^{2}$, Dongwen Zhang ${ }^{2}$, Jinbin Wang ${ }^{1}$, Jianmin Yuan ${ }^{2}$ and Yichun Zhou ${ }^{1}$

Domain orientations and their volume ratios in ferroelectrics are recognized as a compelling topic recently for domain switching dynamics and domain stability in devices application. Here, an optimized second harmonic generation method has been explored for ferroelectric domain characterization. Combing a unique theoretical model with azimuth-polarization-dependent second harmonic generation response, the complex domain components and their distributions can be rigidly determined in ferroelectric thin films. Using the proposed model, the domain structures of rhombohedral $\mathrm{BiFeO}_{3}$ films with $71^{\circ}$ and $109^{\circ}$ domain wall, and, tetragonal $\mathrm{BiFeO}_{3}, \mathrm{~Pb}\left(\mathrm{Zr}_{0.2} \mathrm{Ti}_{0.8}\right) \mathrm{O}_{3}$, and $\mathrm{BaTiO}_{3}$ ferroelectric thin films are analyzed and the corresponding polarization variants are determined. This work could provide a powerful and all-optical method to track and evaluate the evolution of ferroelectric domains in the ferroelectric-based devices.

npj Computational Materials (2018)4:39; doi:10.1038/s41524-018-0095-6

\section{INTRODUCTION}

Ferroelectric materials, possessing excellent ferroelectric and electro-optical (EO) properties, have fostered the development of electronic and optoelectronic devices, e.g., sensors, actuesators, nonvolatile memories, and optical communication systems. ${ }^{1-10}$ In those applications, it is critical to understand ferroelectric domain orientations and domain switching through external stimulation, where the domain orientations have an intense influence on ferroelectric properties and the fluctuation of domain volume fractions due to domain switching or backswitching dominates the efficiency and stability of ferroelectric devices. ${ }^{11-23}$ Therefore, the precise determination of ferroelectric domain orientations and their volume fractions is required not only in view of ferroelectric device engineering but also in the corresponding physics mechanism, such as fatigue and retention.

A lot of efforts have been invested to evaluate domain structures of ferroelectrics. The typical methods including piezoresponse force microscopy (PFM) and transmission electron microscopy (TEM) provide high spatial resolution to visualize the local domain structures. ${ }^{24-29}$ However, they still have some drawbacks in accurately measuring domain volume fractions, especially for the in-plane domains in the disordered multidomain system, where the PFM is only able to sense the domain structures near the material surface rather than the domains underneath, and the TEM is a 'destructive' characterization technique with a complicate process of specimen preparation, which usually presents the domain structures at a local region far from the whole picture of the domains in the sample. ${ }^{30-34}$ The precise determination of the domain distributions in ferroelectrics with complex domain structures in a non-destructive manner has not been achieved. Recent advances have pointed out that the second harmonic generation (SHG) is a promising all-optical approach for probing domain structures in ferroelectrics, as the symmetrical-dependent SHG signals present a unique response to the domain structures, and the adjustable probe size could adapt to the scale of the semiconductor devices. ${ }^{35-49}$ Moreover, this optical approach can readily access the domain structures in the heterostructures that the ferroelectric layer is covered by nonferroelectric materials.

Since the SHG response is highly sensitive to the polarization angle of the incident light with respect to ferroelectric domain orientations, the polarization-dependent SHG signals are usually used to study domain structures. ${ }^{50-53}$ However, in a ferroelectric with many possible domain variants, the quantitative analysis becomes very complex, which makes it difficult to achieve a completed picture for domain structures through the polarizationdependent SHG response only along the fixed domain directions of a ferroelectric sample. . $^{37,42,54,55}$ To solve this problem, an azimuth-dependent SHG is introduced to distinguish the predominant domain orientation from many random variants, because a rotation of a sample around $z$-axis can change the ferroelectric polarization directions of individual domains relative to the incident beam without external electric field, where different oriented domains lead to different SHG responses. Therefore, in this work, we have developed an improved ferroelectric domain characterization technique by collecting azimuth-polarization-dependent SHG signals with varying both the polarization angle of the incident beam and the azimuth angle of sample relative to the beam. By fitting the SHG signals to an established theoretical model, it is possible to correlate the SHG signals to the domain distributions in the measured area. Using this method, we give examples on the rhombohedral $\mathrm{BiFeO}_{3}(\mathrm{BFO})$

\footnotetext{
${ }^{1}$ School of Materials Science and Engineering, Xiangtan University, 411105 Hunan, China; ${ }^{2}$ Department of Physics, College of Science, National University of Defense Technology, 410073 Hunan, China; ${ }^{3}$ Department of Materials Science and Engineering, National Chiao Tung University, 30010 Hsinchu, Taiwan and ${ }^{4}$ Department of Physics and Astronomy, University of Nebraska-Lincoln, Lincoln 68588, USA

Correspondence: Xiangli Zhong (xlzhong@xtu.edu.cn) or Jianmin Yuan (jmyuan@nudt.edu.cn) or Yichun Zhou (zhouyc@xtu.edu.cn)

These authors contributed equally: Yuan Zhang, Yi Zhang.
}

Received: 26 October 2017 Revised: 13 June 2018 Accepted: 28 June 2018

Published online: 24 July 2018 
(a)

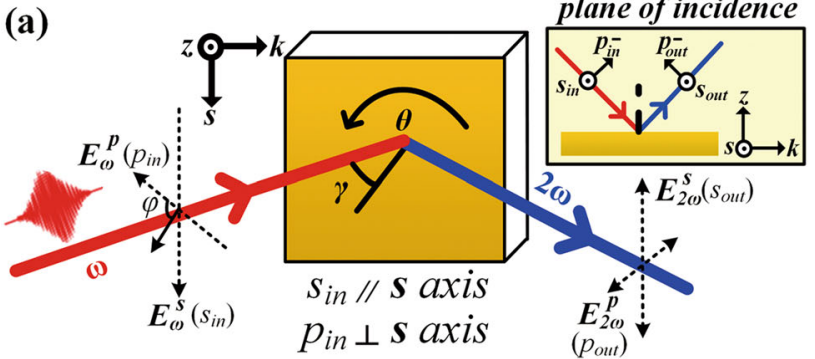

(b)

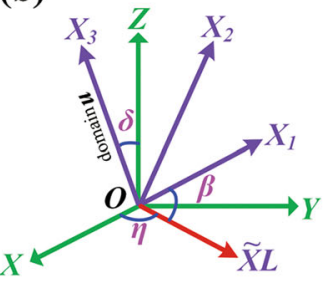

(c)

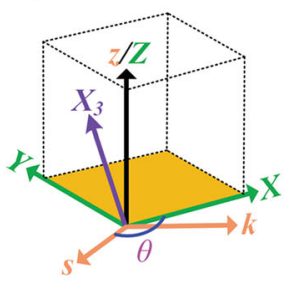

Fig. 1 a Schematic diagram of SHG experimental system, where lab frame $(s, k, z)$ is defined with $s$ axis parallel to the $s_{\text {in }}$ incident beam. $\mathbf{b}$ Schematic sketch of arbitrarily oriented domain variant $u$ in sample coordinate $(X, Y, Z)$. The $\beta$ is the angle between $X_{1}$ axis and the intersection line $(\tilde{X} L)$ of $X-Y$ and $X_{1}-X_{2}$ plane. The $\eta$ is the angle between $X$ axis and line $\tilde{X} L$. The $\delta$ is the angle between $X_{3}$ axis and $Z$ axis. c Schematic sketch of the defined sample coordinate $(X, Y, Z)$ in the lab coordinate $(s, k, z)$, where the $\theta$ is the angle between $s$ and $X$ axes, or, $k$ and $Y$ axes

thin films with $71^{\circ}$ and $109^{\circ}$ domain walls, where the domain orientations and their volume factions are determined based on the collected SHG signals. Moreover, we also apply this SHG method to many other ferroelectric systems for domain structure characterization, such as, tetragonal BFO, $\mathrm{Pb}\left(\mathrm{Zr}_{0.2} \mathrm{Ti}_{0.8}\right) \mathrm{O}_{3}(\mathrm{PZT})$, and $\mathrm{BaTiO}_{3}$ (BTO) thin films.

\section{RESULTS}

The experimental setup of SHG is schematically shown in Fig. 1a. A laser beam is incident on a ferroelectric sample with an incident angle $\gamma\left(\gamma=45^{\circ}\right)$ and the generated SHG signals are collected in the reflection configuration. The linearly polarized fundamental electric-field $E^{\omega}(\varphi)$ of the incident light can be rotated through adjusting the angle $\varphi$ in the range of $0^{\circ}$ to $360^{\circ}$, where $\varphi=0^{\circ}$ and $\varphi=180^{\circ}$ correspond to $p$-polarized light field of $p_{\text {in }}^{+}$and $p_{\text {in }}^{-}$, and, $\varphi$ $=90^{\circ}$ and $\varphi=270^{\circ}$ correspond to $s$-polarized light field of $s_{\text {in }}^{+}$and $s_{\text {in }}^{-}\left(p_{\text {in }}^{+}\right.$and $s_{\text {in }}^{+}$are abbreviated as $p_{\text {in }}$ and $\left.s_{\text {in }}\right)$. The lab frame $(s, k, z)$ is defined with $s$ axis parallel to the direction of $s_{\text {in }}$ light field, and the sample coordinate $(X, Y, Z)$ is defined to describe the direction of sample edges with respect to the optics. The sample can be rotated by a variable angle $\theta$ in the $s-k$ plane, where $\theta=0^{\circ}$ is defined as the initial position when the $X$ axis coincides with the direction of $s_{\text {in }}$ light field. The generated $p$ or $s$ polarized ( $p_{\text {out }}$ and $s_{\text {out }}$ ) SHG signals are selected to detect by an analyzer, and the localized SHG polarimetry system is performed. The nonlinear polarization of SHG can be expressed as $P_{\mathrm{i}}^{2 \omega}=d_{\mathrm{ijk}} E_{\mathrm{j}} E_{\mathrm{k}}$, where $d_{\mathrm{ijk}}$ is the nonlinear optical tensor, and the crystallography coordinate $\left(X_{1}, X_{2}, X_{3}\right)$ is established to denote the orientation of nonlinear optical tensor $\left(d_{\mathrm{ijk}}\right)$ coordinates of each individual domain variants (the direction of each individual domain variants is along the $X_{3}$ axis). An assumed arbitrarily oriented domain variant $u$ is defined by three Euler angles $(\eta, \beta, \delta)$ in the sample coordinate $(X, Y, Z)$ as shown in Fig. $1 \mathrm{~b}$, and the sample coordinate $(X, Y, Z)$ is defined in the lab coordinate $(s, k, z)$ by angle $\theta$ as shown in Fig. 1c. The nonlinear polarizations for a domain $u$ are calculated by transforming the nonlinear tensor to the lab frame $(s, k, z)$ with the rule $T_{\mathrm{ijk}}^{\text {new }}=a_{\mathrm{il}} a_{\mathrm{jm}} a_{\mathrm{kn}} T_{\mathrm{Imn}}^{\text {old }}$, where $T$ is the third rank property tensor, and $a_{i l}=e_{i}^{\text {new }} \cdot \hat{e}_{i}^{\text {olldn }}(i, l=1,2,3)$ is the corresponding coordinate transformation matrix. The calculated nonlinear polarization components $P_{\mathrm{i}}^{2 \omega, \mathrm{u}}$ (along $i$ direction) are determined by the angle $\eta, \delta, \varphi, \beta, \gamma$, and $\theta$, where the details of the above calculations are shown in the Supplementary Materials. Moreover, the signals generated by domain wall are mostly negligible in the analysis due to its much smaller relative area fraction compared with domains. Therefore, the $p_{\text {out }}$ and $s_{\text {out }} \mathrm{SHG}$ electric fields $E_{(\mathrm{p}, \mathrm{u})}^{2 \omega, \mathrm{u}}$ generated by a domain variant $u$ can be defined as following:

$E_{\mathrm{p}}^{2 \omega, \mathrm{u}}=\left|S_{\mathrm{p}}\left(F_{\mathrm{s}} P_{\mathrm{z}}^{2 \omega, \mathrm{u}}-F_{\mathrm{c}} P_{\mathrm{k}}^{2 \omega, \mathrm{u}}\right)\right|$,

$E_{\mathrm{s}}^{2 \omega, \mathrm{u}}=\left|S_{\mathrm{s}} P_{\mathrm{s}}^{2 \omega, \mathrm{u}}\right|$
The meanings of $S_{\mathrm{p}}, S_{\mathrm{s}}, F_{\mathrm{s}}$, and $F_{\mathrm{c}}$ are shown in the Supplementary Materials. The total SHG intensity $l_{(\mathrm{p}, \mathrm{s})}^{2 \omega}$ contributed from all the individual domains can be expressed as:

$I_{(\mathrm{p}, \mathrm{s})}^{2 \omega}=\left|E_{(\mathrm{p}, \mathrm{s})}^{2 \omega}\right|=\left|\sum F_{\mathrm{u}} E_{(\mathrm{p}, \mathrm{s})}^{2 \omega, \mathrm{u}}\right|^{2}+b_{(\mathrm{p}, \mathrm{s})}$.

$F_{\mathrm{u}}$ is the fraction ratio of the domain variant $u$ and the $b_{(\mathrm{p}, \mathrm{s})}$ is artificially added to simulate the background. Based on the Eq. (3), we can know that the SHG intensity increases with the increasing of volume fraction $F_{\mathrm{u}}$, and the total SHG electric field $E_{(\mathrm{p}, \mathrm{s}}^{2 \omega}$ originates from the superposition of fields from all individual domains, where the " + " and "-" fields are compensated. Here, the positive oriented of in-plane (IP) and out-of-plane (OP) domain components are defined: along $s$ and $-k$, and, $-z$ axes, respectively. According to the above model, some criterions can be established. Firstly, the IP domain components can be distinguished by analyzing the azimuth-polarization-dependent SHG patterns, because the changes of SHG patterns caused by sample rotation are attributed to the IP domain components. Secondly, the OP domain components can be verified by comparing with the SHG patterns at $\theta=0^{\circ}$ and $180^{\circ}$, because the IP domain components give an opposite sign of SHG fields with same magnitude after a sample rotation by $180^{\circ}$, if the sample has no OP domain components, the SHG patterns should be same when the $\theta$ equals $0^{\circ}$ and $180^{\circ}$. Thirdly, the vertical relationship between IP domain components and $s_{\text {in }}$ light filed can be distinguished by observing the $s_{\text {in }}-s_{\text {out }}$ SHG signals, since the domain components do not contribute to $s_{\text {in }}-s_{\text {out }}$ SHG signals when they are perpendicular to the direction of $s_{\text {in }}$ light filed (the OP domain components always do not generate $s_{\text {in }}-s_{\text {out }}$ signals, and the details are shown in Supplementary Materials). In our experiments, only the $s_{\text {out }}$ SHG signals are selected as an output, and the domain distributions can be figured out by fitting the measured $s_{\text {out }}$ SHG intensity.

\section{DISCUSSION}

The BFO thin films are chosen as a model system in our work. This films have four structural variants lied along $<111>$ directions with eight distinct polarization variants on the $(001)_{C}$ perovskite surface (Fig. 2a). The $r_{1}, r_{2}, r_{3}$, and $r_{4}$ correspond to $P_{1}^{++}, P_{2}^{+/-}, P_{3}^{+/-}$, and $P_{4}^{+}+$, respectively, where the "+" and "-" stand for "up" and "down" polarization directions. ${ }^{57-60}$ The allowed polarization configurations can form domain boundaries along either $\{101\}_{C}$ or $\{100\}_{C}$, so called $71^{\circ}$ or $109^{\circ}$ domain walls, respectively. Firstly, the BFO film with $71^{\circ}$ domain wall is studied by SHG. The IP-PFM image of this thin film is shown in Fig. 2b, which exhibits the ordered $71^{\circ}$ domain arrays. ${ }^{59}$ The possible variant patterns of BFO film with $71^{\circ}$ domain wall are (011) $-r_{1} / r_{2},(\overline{10} \overline{1})-r_{2} / r_{3},(0 \overline{11})-r_{3} / r_{4}$ and (101)- $r_{4} / r_{1} .{ }^{58}$ The projected polarizations of $P_{1}^{-}, P_{2}^{-}, P_{3}^{-}$, and $P_{4}^{-}$ are shown by the sense of arrows in Fig. $2 c$ (the sample coordinate axes are defined as $X=[100]_{\mathrm{p}}, \quad Y=[010]_{\mathrm{p}}$, and $Z=[001]_{\mathrm{p}}$ directions of the BFO film, respectively). The polarization- 
(a)

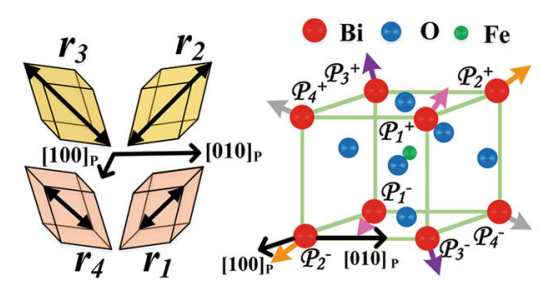

(b)

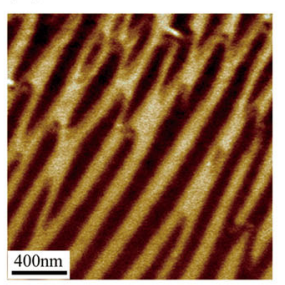

(c)

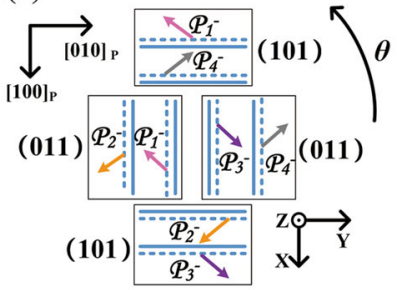

(d)

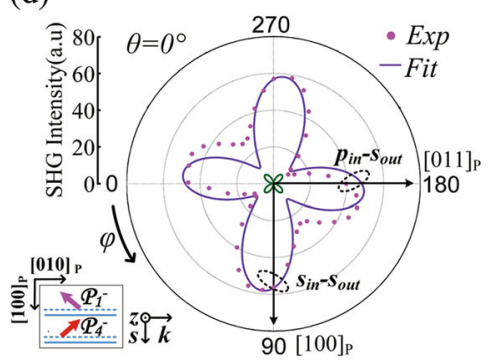

(g)

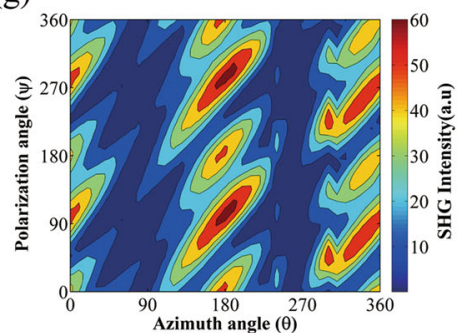

(e)

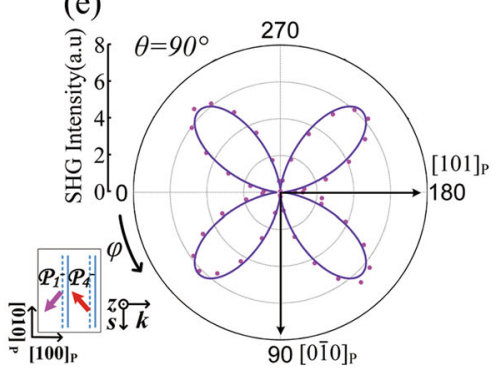

(h)

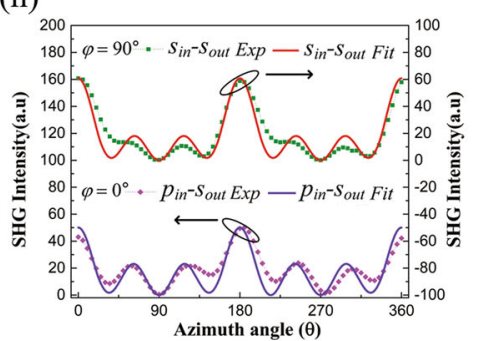

(f)

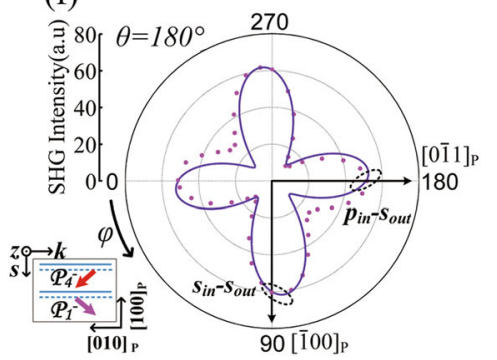

(i)

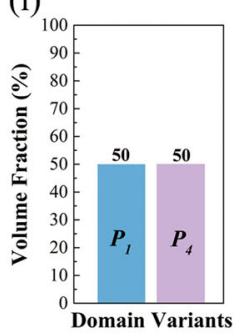

Fig. 2 a Schematic of four different structural variants and eight domain variants in (001) rhombohedral BFO film. $\mathbf{b}$ IP-PFM image of the BFO thin film with $71^{\circ}$ domain wall. c Possible domain patterns for BFO film with $71^{\circ}$ domain wall. The projected polarizations of the $P_{1}^{-}, P_{2}^{-}, P_{3}^{-}$, and $P_{4}^{-}$are shown by the sense of arrows. d-f Polar plots of measured s-polarized SHG intensity as a function of polarization angle $\varphi$ with $\theta=0^{\circ}$, $90^{\circ}$, and $180^{\circ}$, respectively. $\mathbf{g}$ Contour pattern of monitored s-polarized SHG intensity vs. sample rotation angle $\theta$ and polarization angle $\varphi$. $\mathbf{h}$ The $s$-polarized SHG intensity as a function of angle $\theta$ under the fixed $p_{\text {in }}$ and $s_{\text {in }}$ incident light fields. i Fractions of domain variants in the BFO thin film with $71^{\circ}$ domain wall. The symbols present the experimental data, and the lines indicate the theoretical fits. Comparison of the polarization-dependent SHG patterns with $\theta=0^{\circ}$ and $90^{\circ}$ are as insets in d, where the green is the SHG pattern at $\theta=90^{\circ}$. The schematic illustrations of the domain variants at angle $\theta=0^{\circ}, 90^{\circ}$, and $180^{\circ}$ relative to incident beam are as insets

dependent SHG patterns with $\theta=0^{\circ}, 90^{\circ}$, and $180^{\circ}$ of BFO film with $71^{\circ}$ domain wall are shown in Fig. $2 \mathrm{~d}$-f, respectively. All the SHG patterns present a shape of two double lobes, and the pattern shape changes with the rotation of angle $\theta$. Therefore, the BFO film with $71^{\circ}$ domain wall has IP domain components. After a sample rotation by $180^{\circ}$, one can see that the SHG pattern in Fig. $2 f$ is different from that in Fig. $2 d$, which indicates the existence of OP domain components in BFO film with $71^{\circ}$ domain wall. Moreover, strong $s_{\text {in }}-s_{\text {out }}$ SHG signals can be found in Fig. $2 \mathrm{~d}$ when the $\theta$ equals to $0^{\circ}$, while the $s_{\text {in }}-s_{\text {out }}$ signals disappear in Fig. $2 \mathrm{e}$ when the $\theta$ equals to $90^{\circ}$. In fact, the $s_{\text {in }}-s_{\text {out }}$ SHG signals at the $\theta$ $=0^{\circ}$ are only generated by the IP domain components of $[100]_{p}^{+/-}$ $\left([100]_{p}\right.$ or $\left.[100]_{p}\right)$, since the domain components of $[010]_{p}^{+/}$are perpendicular to the $s_{\text {in }}$ light field in this case. Therefore, the IP domain components could be only reasonably existed along $[100]_{\mathrm{p}}^{+/-}$. Accordingly, the possible domain variants in the BFO film with $71^{\circ}$ domain wall are $r_{2} / r_{3}$ and $r_{4} / r_{1}$.

To further investigate the domain variants of BFO film with $71^{\circ}$ domain wall, the azimuth-polarization-dependent SHG signals are collected at intervals of $10^{\circ}$ in the range of $0^{\circ}$ to $360^{\circ}$. The SHG intensity vs. the angle $\varphi$ and $\theta$ are presented in the contour pattern in Fig. 2g. The maximum of SHG intensity at different polarization angles $\varphi$ under a certain azimuth angle $\theta$ are defined

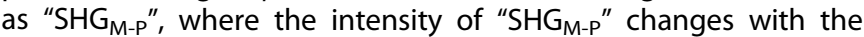
azimuth angle $\theta$. It can be found that the maxima intensity of
"SHG $\mathrm{M}_{\mathrm{P}} \mathrm{P}$ " is present at $\theta=180^{\circ}$, and the minima value is located at $\theta=90^{\circ}$ (the comparison of the polarization-dependent SHG patterns with $\theta=90^{\circ}$ and $\theta=270^{\circ}$ is shown in the Supplementary Materials, Fig. S1). The intensity of "SHG $\mathrm{M}_{\mathrm{P}}$ " is broadly larger at $\theta$ $=\theta_{0}+180^{\circ}\left(\theta_{0}=0^{\circ} \sim 180^{\circ}\right)$ than that at $\theta=\theta_{0}$, where the

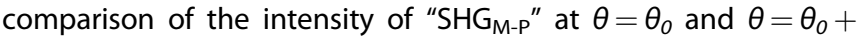
$180^{\circ}$ are presented in the Supplementary Materials (Fig. S2). As a matter of the fact, the intensity of "SHG ${ }_{M-P}$ " would be enlarged after a rotation of sample by $180^{\circ}$, when the signs of the SHG fields from net IP and OP domain components are opposite at initial states, otherwise, the SHG intensity would be decreased. Hence, we can infer that the signs of the SHG fields from net IP and OP domain components of BFO film with $71^{\circ}$ domain wall is opposite at $\theta=\theta_{0}$, which exhibits as: "+" and "-", or, "-" and "+". Therefore, the orientations of net IP and OP polarizations are along $[100]_{p}$ and $[001]_{p}$, or, $[\overline{100}]_{p}$ and $[00 \overline{1}]_{p}$. Summing up, the possible domain variants in the BFO film with $71^{\circ}$ domain wall are $r_{4} / r_{1}$. Then, we extract the SHG signals as a function of angle $\theta$ under the fixed $p_{\text {in }}$ and $s_{\text {in }}$ incident light fields, and the results of $p_{\text {in }}-s_{\text {out }}$ and $s_{\text {in }}-s_{\text {out }}$ are shown in Fig. $2 i$. The line profiles have the same varying tendency and the intensity peaks are located at $\theta=$ $n^{*} 180^{\circ}(n=0,1,2)$, while the valley positions are located at $\theta=$ $90^{\circ}+n^{*} 180^{\circ}(n=0,1)$. We know that the SHG intensity from the OP domain components is fixed under the $p_{\text {in }}-s_{\text {out }}$ geometry, and it is not existed under the $s_{\text {in }}-s_{\text {out }}$ geometry, thus the peak signals 
(a)

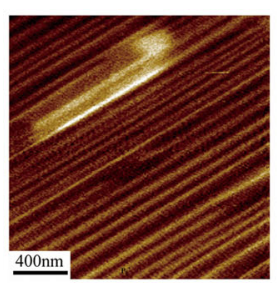

(d)

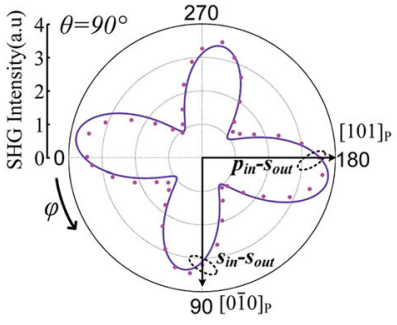

(g)

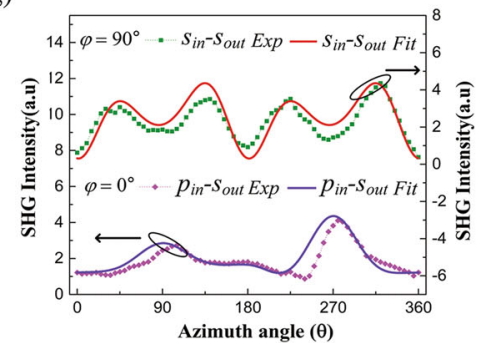

(b)

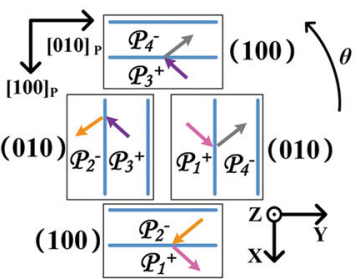

(e)
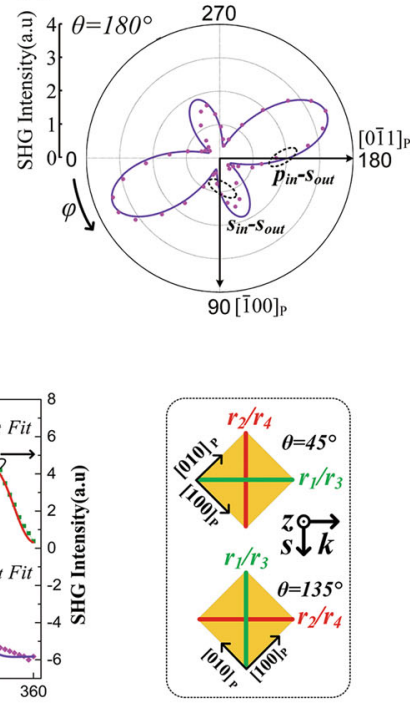

(c)

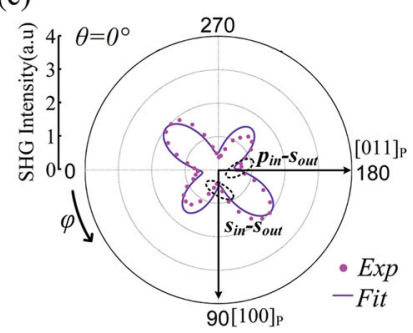

(f)

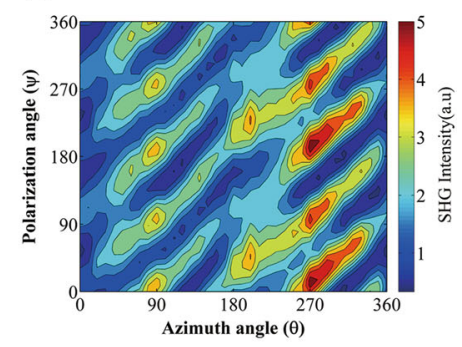

(h)

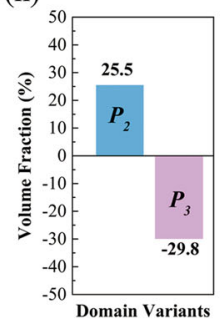

Fig. 3 a IP-PFM image of the BFO thin film with $109^{\circ}$ domain wall. b Possible domain patterns for BFO film with $109^{\circ}$ domain wall. The projected polarizations of the $P_{1}^{+}, P_{2}^{-}, P_{3}^{+}$, and $P_{4}^{-}$are shown by the sense of arrows. c-e Polarization-dependent s-polarized SHG patterns with $\theta=0^{\circ}, 90^{\circ}$, and $180^{\circ}$, respectively. f Contour pattern of monitored s-polarized SHG intensity vs. angles $\theta$ and $\varphi$. g Azimuth-dependent SHG intensity under the $p_{\mathrm{in}}-s_{\text {out }}$ and $s_{\mathrm{in}}-s_{\text {out }}$ geometry. $\mathbf{h}$ Fractions of domain variants in BFO thin film with $109^{\circ}$ domain wall. The schematic illustrations of the domain variants at $\theta=45^{\circ}$ and $\theta=135^{\circ}$ with respect to incident light are as insets

are mostly attributed to IP domain components of $[100]_{p}^{+/-}$at $\theta=$ $n^{*} 180^{\circ}(n=0,1,2)$. Therefore, those azimuth-dependent SHG intensity features further illustrate that the net IP domain components are only along $[100]_{\mathfrak{p}}^{+/-}$directions. Moreover, the volume fractions of the net polarization vectors are analyzed by fitting the measured azimuth-polarization-dependent SHG signals by using Eqs. (2) and (3) (the results are shown in Fig. 2i), where the volume fractions of net polarization vector $\left(P_{i}\right)$ is defined as: the differences of the fractions of the positive $\left(P_{\mathrm{i}}^{+}\right)$and negative ( $P_{i}^{-}$) domains. The BFO film with $71^{\circ}$ domain wall possesses the net polarization vectors of $P_{4}$ and $P_{1}$ with both volume fractions of $50 \%$. In addition, an estimation of domain fraction was made by contrast statistics based on vector PFM (Supplementary Fig. S6 and Fig. S7), which is agreement with our SHG results.

The BFO film with $109^{\circ}$ domain wall is also studied by SHG. The IP-PFM image exhibits the ordered $109^{\circ}$ domain arrays (Fig. 3a). ${ }^{60}$ The possible variants for BFO film with $109^{\circ}$ domain wall are (100)$r_{1} / r_{2},(010)-r_{2} / r_{3},(100)-r_{3} / r_{4}$, and (010)- $r_{4} / r_{1},{ }^{58}$ and the projected polarizations of the $P_{1}^{+}, P_{2}^{-}, P_{3}^{+}$and $P_{4}^{-}$are shown by the sense of arrows in Fig. 3b. Figure 3c-e shows the polarization-dependent SHG patterns with $\theta=0^{\circ}, 90^{\circ}$, and $180^{\circ}$, respectively. The SHG pattern shape and signal intensity are in greatly differences, which implies the existence of both IP and OP domain components. Meanwhile, we can see that strong $s_{\text {in }}-s_{\text {out }}$ SHG signals both present in Fig. 3c, d, which suggests the existence of net IP domain components along $[100]_{\mathfrak{p}}^{+/-}$and $[010]_{\mathfrak{p}}^{+/-}$. Moreover, the intensity of $s_{\text {in }}{ }^{-} s_{\text {out }}$ signals in Fig. $3 d$ is significantly higher than that in Fig. 3c, which means that the net IP domain population of $[010]_{p}^{+/-}$should be more than that of $[100]_{p}^{+/-}$. Therefore, the possible variant patterns of BFO film with $109^{\circ}$ domain wall are $r_{2} /$ $r_{3}$ and $r_{4} / r_{1}$. The SHG intensity vs. the angle $\varphi$ and $\theta$ is shown in Fig.

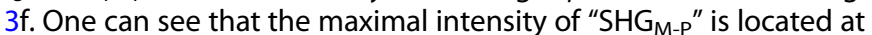
$\theta=270^{\circ}$, and the intensity of "SHG $\mathrm{M}_{-\mathrm{P}}$ " at $\theta=\theta_{0}+180^{\circ}$ is obviously stronger than that at $\theta=\theta_{0}$ (the change process of the intensity of "SHG $\mathrm{M}_{\mathrm{P}}$ " at $\theta=\theta_{0}$ and $\theta=\theta_{0}+180^{\circ}$ are shown in the Supplementary Materials, Fig. S2), which suggest that the signs of SHG fields from the largest population of net IP and OP domain components are opposite at initial states. To further determine the signs of fields from the two IP domain components, the azimuth-dependent SHG response of $p_{\text {in }}-s_{\text {out }}$ are analyzed. In Fig. $3 \mathrm{~g}$, the line profile presents two peaks at $\theta=90^{\circ}$ and $270^{\circ}$, and the maxima is located at $270^{\circ}$. We can know that the maximal SHG intensity of $p_{\text {in }}-s_{\text {out }}$ occurs only when the sign of the fields from all IP and OP domain components are at same under $p_{\text {in }}$ light field. Thus, the sign of the fields from two IP domain components are in the same at $\theta=270^{\circ}$, while they are opposite at $\theta=0^{\circ}$. Therefore, the orientations of two net IP and one OP polarizations are along $[\overline{100}]_{p^{\prime}}[0 \overline{1} 0]_{p^{\prime}}$ and $[001]_{p}$, or, $[100]_{p},[010]_{p}$, and $[00 \overline{1}]_{p^{\prime}}$ where the signs of the fields exhibit as: "-", "+", and "-", or, " $+{ }^{\prime \prime}$, "-", and "+". In this case, the possible domain variants of $P_{4}^{-} / P_{1}^{+}$ and $P_{4}^{+} / P_{1}^{-}$can be excluded in the BFO film with $109^{\circ}$ domain. This conclusion is further supported by the $s_{\text {in }}-s_{\text {out }}$ SHG signals as shown in Fig. 3g, where the line profile presents four peaks at $\theta=$ $45^{\circ}+n^{*} 180^{\circ}(n=0,1)$ and $\theta=135^{\circ}+n^{*} 180^{\circ}$. In fact, when the $\theta$ $=45^{\circ}+n^{*} 180^{\circ}$, the $s_{\text {in }}-s_{\text {out }}$ SHG signals are only generated by IP projections of domain $P_{i+2}^{+}(i=0,2)$, because the IP projections of 
(a)

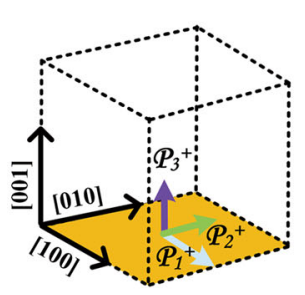

(b)

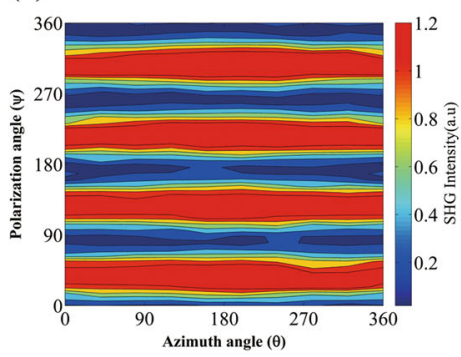

(c)

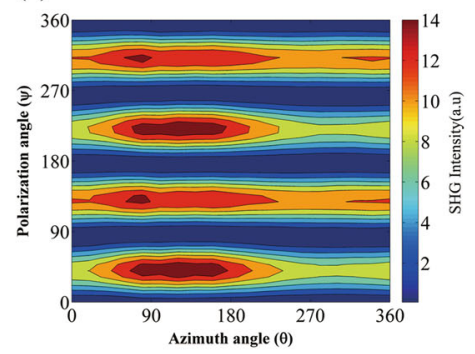

(d)

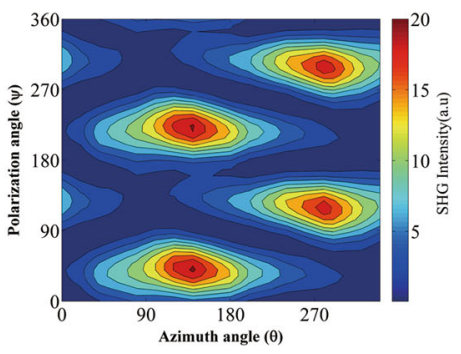

(e)

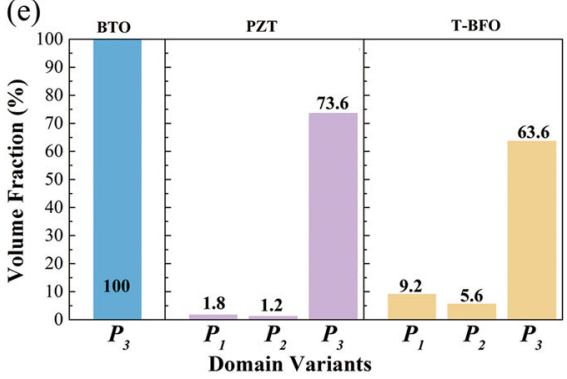

Fig. 4 a Schematic of domain variants of (001)-oriented tetragonal BTO, PZT, and BFO thin films. Contour pattern of s-polarized monitored SHG intensity vs. the angles $\theta$ and $\varphi$, b BTO, c PZT, and d BFO films. e Fractions of domain variants in the BTO, PZT, and BFO thin films

domain $P_{i+1}^{+/-}$are perpendicular to the $s_{i n}$ light field in this case. In contrast, when the $\theta=135^{\circ}+n^{*} 180^{\circ}$, the $s_{\text {in }} s_{\text {out }}$ signals are only generated by IP projections of domain $P_{i+1}^{+1-}$, since the IP projections of domain $P_{i+2}^{+/-}$are perpendicular to the $s_{\text {in }}$ light field in that case (the schematic illustrations of the domain variants at $\theta=45^{\circ}$ and $\theta=135^{\circ}$ with respect to incident light are shown as insets in Fig. 3g). Thus, the larger SHG intensity at $\theta=135^{\circ}+$ $n^{*} 180^{\circ}$ suggests that the population of the net IP polarizations from $P_{i+1}$ are more than that from $P_{i+2}$ in the BFO film with $109^{\circ}$ domain wall. Accordingly, the possible domain variants of the BFO film with $109^{\circ}$ domain wall are $r_{2} / r_{3}$, where the population of the net ferroelectric polarization from $P_{3}$ are larger than that from $P_{2}$. By fitting the measured SHG signals, the volume fractions of net $P_{2}$ and $P_{3}$ in the BFO film with $109^{\circ}$ domain wall can be determined, i.e., $25.5 \%$ and $-29.8 \%$, respectively (Fig. $3 \mathrm{~h}$ ), and a further analysis of the domain variants in this BFO film are presented in the Supplementary Materials. Moreover, by correlating the BFO domain structures and contour patterns shown in Figs. $2 \mathrm{~g}$ and $3 f$, we can draw the conclusions that the inclined stripes are attributed to the rhombohedral phase structures in the BFO films. The BFO film with $71^{\circ}$ domain wall exhibits larger SHG intensity than that in the BFO with $109^{\circ}$ domain wall, due to the larger population of net IP and OP polarizations in the BFO film with $71^{\circ}$ domain wall. The stripes of the contour pattern in the BFO film with $71^{\circ}$ domain wall are "discontinuous", while they are "continuous" in the BFO film with $109^{\circ}$ domain wall, this results are largely determined by the net IP domain components in the BFO films where the BFO film with $71^{\circ}$ domain wall only has one direction of net IP domain components along $[100]_{\mathrm{p}}^{+/-}$, but there are two directions of net IP domain components along $[100]_{p}^{+/-}$ and $[010]_{\mathfrak{p}}^{+/-}$for BFO film with $109^{\circ}$ domain wall in our experiments. The calculated contour pattern of s-polarized SHG intensity vs. angles $\theta$ and $\varphi$ was calculated based on the established azimuth-polarization-dependent model (Supplementary Fig. S8), which further support the above conclusions.

To further illustrate the relationship between azimuthpolarization-dependent SHG signals and domain variants, the typical tetragonal (001)-oriented BTO, PZT, and BFO are also investigated. The possible domain variants of (001)-oriented BTO, PZT, and T-BFO are shown in Fig. $4 a$, where those films have six polarization orientations of $P_{1}^{+\gamma-}, P_{2}^{+/-}$, and $P_{3}^{+/-}$. Their SHG intensity as a function of angle $\varphi$ and $\theta$ are presented in Fig. 4b-d, respectively. The domain orientations and their corresponding volume fractions are measured, and the results are shown in Fig. 4e. It can be seen that the BTO film only has OP domains, and the PZT and BFO films have majority OP domains and minority IP domains, while the BFO film shows more population of IP domains than that in the PZT film. By observing the SHG contour patterns in Fig. $4 a-c$, we can find that all the stripes in SHG pattern are horizontal, and the stripes are continuous and invariant from BTO film, while they are continuous but not uniform from PZT film and they are partial discontinuous from BFO film. Based on that, we can deduce that the tetragonal (001)-oriented ferroelectric system exhibits horizontal stripes, and the stripes change from continuity to discontinuity when the population of net IP domains are increasing gradually. We note that the SHG method has resolution limitation comparing with PFM techniques, and its resolution is in the order of hundreds of nanometers. However, the SHG also exhibits the advantages of the adjustable spot size, which can be used for the characterization of ferroelectric devices.

In summary, we report an optimized SHG technique for ferroelectric domain characterizing, in which the correlation between SHG response and domain distributions are established. Based on this all-optical SHG method, all the domain variants and its population in BFO ferroelectric films are determined. We also apply our method to many other perovskite ferroelectrics, i.e., TPZT, T-BTO, and T-BFO. This work provides an effective and alloptical method to determine the domain distribution for ferroelectric thin films and devices including the field of multifunctional heterostructures which combine with functional metals and complex oxide thin films.

\section{METHODS}

The (001)-oriented rhombohedral (R-phase) $\mathrm{BiFeO}_{3}$ (BFO) films of approximately $60 \mathrm{~nm}$ thick with $71^{\circ}$ and $109^{\circ}$ domain wall were grown on $\mathrm{SrRuO}_{3}$-buffered and no-buffered $\mathrm{DyScO}_{3}(110)$ substrates by pulsed laser deposition (PLD), respectively. The (001)-oriented tetragonal (T-phase) $\mathrm{BaTiO}_{3}$ (BTO, $20 \mathrm{~nm}$, grown on $\mathrm{SrRuO}_{3}$-buffered $\mathrm{SrTiO}_{3}$ substrate), BFO ( $40 \mathrm{~nm}$, grown on $\mathrm{LaNiO}_{3}$-buffered $\mathrm{LaAlO}_{3}$ substrate) and $\mathrm{Pb}\left(\mathrm{Zr}_{0.2} \mathrm{Ti}_{0.8}\right) \mathrm{O}_{3}$ (PZT, $150 \mathrm{~nm}$, grown on $\mathrm{SrRuO}_{3}$-buffered $\mathrm{SrTiO}_{3}$ substrate) were also prepared by PLD. The domain structures were characterized by commercially available AFM platform (Dimension Icon AFM, Bruker) and 
the crystalline structure were characterized via X-ray Diffraction (Rigaku-D/ Max 2500) with the Cu Ka radiation.

The SHG measurements were performed by using $800 \mathrm{~nm}$ laser $(150 \mathrm{fs}$, $10 \mathrm{~nJ}, 76 \mathrm{MHz}$ ) generated by a coherent Ti:sapphire pulsed laser as the fundamental laser beam. This fundamental laser beam was focused by a long-focus lens onto the sample with a focal spot diameter of $\sim 100 \mu \mathrm{m}$ and the incident peak intensity is $\sim 10 \mathrm{MW} / \mathrm{cm}^{2}$ at the sample position. The generated SHG signals from the sample were detected by a photomultiplier tube (PMT) with a fixed incident angle of $45^{\circ}\left(\gamma=45^{\circ}\right)$ in the reflection geometry. The long-pass filter was located in front of the sample to pass through the fundamental laser beam, and the bandpass interference filter was installed behind the sample to filter out the fundamental laser beam. The Glan polarizer was rotated to adjust and analyze the generated SHG signals from the sample at $0^{\circ}$ and $90^{\circ}$ configurations, which corresponds to $p$-polarized ( $p$-out) and $s$-polarized ( $s$ out) SHG signals, respectively.

\section{Data availability}

All data needed to evaluate the conclusions in the paper are present in the paper and/or the Supplementary Materials. Additional data related to this paper may be requested from the authors.

\section{ACKNOWLEDGEMENTS}

This work was financially supported by the National Natural Science Foundation of China $(51572233,61574121,11372266$ and 11474359) and the National Key Research and Development Program of China (2016YFB0501303).

\section{AUTHOR CONTRIBUTIONS}

Y.Z., X.Z., J.Y., and Y.Z. conceived the concept. Y.Z. carried out all SHG measurements. Y.Z., Q.G., D.Z., Z.L., and J.Y. built and discussed the fitted models of SHG. G.Z., J.J., C.T., and M.L. prepared all ferroelectric samples and characterized their domain structures. Y.C., H.L., Y.Z., and J.W. analyzed the PFM results of all samples. Y.Z., Y.Z., X.Z., J.Y., and $Y . Z$. analyzed all data and co-wrote the manuscript. All the authors edited, discussed, and approved the whole paper.

\section{ADDITIONAL INFORMATION}

Supplementary information accompanies the paper on the npj Computational Materials website (https://doi.org/10.1038/s41524-018-0095-6).

Competing interests: The authors declare no competing interests.

Publisher's note: Springer Nature remains neutral with regard to jurisdictional claims in published maps and institutional affiliations.

\section{REFERENCES}

1. $\mathrm{Xu}, \mathrm{R}$. et al. Ferroelectric polarization reversal via successive ferroelastic transitions. Nat. Mater. 14, 79-86 (2015).

2. Lee, Y. et al. Bifunctional non-noble metal oxide nanoparticle electrocatalysts through lithium-induced conversion for overall water splitting. Nat. Commun. 6, 1-8 (2015).

3. Scott, J. F. Applications of modern ferroelectrics. Science 315, 954-959 (2007).

4. Setter, N. et al. Ferroelectric thin films: review of materials, properties and applications. J. Appl. Phys. 100, 051606 (2006).

5. Muralt, P. Ferroelectric thin films for micro-sensors and actuators: a review. J. Micromech. Microeng. 10, 136-146 (2000).

6. Eom, C. B. \& Trolier-McKinstry, S. Thin-film piezoelectric MEMS. Mrs. Bull. 37, 1007-1017 (2012).

7. Huang, C. \& Chen, L. Negative poisson's ratio in modern functional materials. Adv. Mater. 28, 8079-8096 (2016).

8. Chanthbouala, A. et al. Solid-state memories based on ferroelectric tunnel junctions. Nat. Nanotechnol. 7, 101-104 (2012).

9. Xiong, C. et al. Active silicon integrated nanophotonics: ferroelectric $\mathrm{BaTiO}_{3}$ devices. Nano. Lett. 14, 1419-1425 (2014).

10. Wessels, B. W. Ferroelectric epitaxial thin films for integrated optics. Annu. Rev. Mater. Res. 37, 659-679 (2007)

11. Dawber, M., Rabe, K. M. \& Scott, J. F. Physics of thin-film ferroelectric oxides. Rev. Mod. Phys. 77, 1083-1130 (2005).

12. Scott, J. F. Searching for new ferroelectrics and multiferroics: a user's point of view. npj Comput. Mater. 1, 15006 (2015).
13. Rogan, R. C., Tamura, N., Swift, G. A. \& Üstündag, E. Direct measurement of triaxial strain fields around ferroelectric domains using X-ray microdiffraction. Nat. Mater. 2, 379-381 (2003).

14. Wang, P. et al. Photo-induced ferroelectric switching in perovskite $\mathrm{CH}_{3} \mathrm{NH}_{3} \mathrm{Pbl}_{3}$ films. Nanoscale 9, 3806-3817 (2017).

15. Otto, T., Grafström, S., Chaib, H. \& Eng, L. M. Probing the nanoscale electro-optical properties in ferroelectrics. Appl. Phys. Lett. 84, 1168-1170 (2004).

16. Lu, W., Fang, D. N., Li, C. Q. \& Hwang, K. C. Nonlinear electric-mechanical behavior and micromechanics modelling of ferroelectric domain evolution. Acta Mater. 47, 2913-2926 (1999).

17. Li, Y. L., Hu, S. Y., Liu, Z. K. \& Chen, L. Q. Effect of substrate constraint on the stability and evolution of ferroelectric domain structures in thin films. Acta Mater. 50, 395-411 (2002).

18. Huo, Y. Z. \& Jiang, Q. Modeling of domain switching in polycrystalline ferroelectric ceramics. Smart Mater. Struct. 6, 441-447 (1997).

19. Jiang, A. Q., Lee, H. J., Hwang, C. S. \& Scott, J. F. Sub-Picosecond processes of ferroelectric domain switching from field and temperature experiments. Adv. Funct. Mater. 22, 192-199 (2012).

20. Speck, J. S., Seifert, A., Pompe, W. \& Ramesh, R. Domain configurations due to multiple misfit relaxation mechanisms in epitaxial ferroelectric thin films. II. Experimental verification and implications. J. Appl. Phys. 76, 477-483 (1994).

21. Pertsev, N. A. \& Zembilgotov, A. G. Domain populations in epitaxial ferroelectric thin films: theoretical calculations and comparison with experiment. J. Appl. Phys. 80, 6401-6406 (1996).

22. Wu, A., Vilarinho, P. M., Shvartsman, V. V., Suchaneck, G. \& Kholkin, A. L. Domain populations in lead zirconate titanate thin films of different compositions via piezoresponse force microscopy. Nanotechnology 16, 2587-2595 (2005).

23. Yuan, G. et al. Ferroelectric domain evolution with temperature in $\mathrm{BaTiO}_{3}$ film on (001) $\mathrm{SrTiO}_{3}$ substrate. Appl. Phys. Lett. 103, 062903 (2013).

24. Gruverman, A. \& Kalinin, S. V. Piezoresponse force microscopy and recent advances in nanoscale studies of ferroelectrics. J. Mater. Sci. 41, 107-116 (2006).

25. Peter, F., Rudiger, A., Szot, K., Waser, R. \& Reichenberg, B. Sample-tip interaction of piezoresponse force microscopy in ferroelectric nanostructures. IEEE. T. Ultrason. Ferr. 53, 2253-2260 (2006).

26. Ding, Y., Liu, J. S. \& Wang, Y. N. Transmission electron microscopy study on ferroelectric domain structure in $\mathrm{SrBi}_{2} \mathrm{Ta}_{2} \mathrm{O}_{9}$ ceramics. Appl. Phys. Lett. 76, 103-105 (2000).

27. Eng, L. M., Güntherodt, H. J., Schneider, G. A., Köpke, U. \& Muñoz Saldaña, J. Nanoscale reconstruction of surface crystallography from three-dimensional polarization distribution in ferroelectric barium-titanate ceramics. Appl. Phys. Lett. 74, 233-235 (1999).

28. Laanait, N., Zhang, Z. \& Schlepütz, C. M. Imaging nanoscale lattice variations by machine learning of x-ray diffraction microscopy data. Nanotechnology 27, 374002 (2016).

29. Vasudevan, R. K. et al. Field enhancement of electronic conductance at ferroelectric domain walls. Nat. Commun. 8, 1318 (2017).

30. Kalinin, S. V. \& Bonnell, D. A. Imaging mechanism of piezoresponse force microscopy of ferroelectric surfaces. Phys. Rev. B 65, 125408 (2002).

31. Wang, Z., Suryavanshi, A. P. \& Yu, M. F. Ferroelectric and piezoelectric behaviors of individual single crystalline $\mathrm{BaTiO}_{3}$ nanowire under direct axial electric biasing. Appl. Phys. Lett. 89, 082903 (2006).

32. Kawagishi, T., Kato, A., Hoshi, Y. \& Kawakatsu, H. Mapping of lateral vibration of the tip in atomic force microscopy at the torsional resonance of the cantilever. Ultramicroscopy 91, 37-48 (2002).

33. Peter, F., Rüdiger, A., Waser, R., Szot, K. \& Reichenberg, B. Comparison of in-plane and out-of-plane optical amplification in AFM measurements. Rev. Sci. Instrum. 76, 046101 (2005)

34. Winkler, C. R. et al. Accessing intermediate ferroelectric switching regimes with time-resolved transmission electron microscopy. J. Appl. Phys. 112, 052013 (2012).

35. Denev, S. A., Lummen, T. T. A., Barnes, E., Kumar, A. \& Gopalan, V. Probing ferroelectrics using optical second harmonic generation. J. Am. Ceram. Soc. 94, 2699-2727 (2011).

36. Kumar, A. et al. Probing mixed tetragonal/rhombohedral-like monoclinic phases in strained bismuth ferrite films by optical second harmonic generation. Appl. Phys. Lett. 97, 112903 (2010).

37. Trassin, M., Luca, G. D., Manz, S. \& Fiebig, M. Probing ferroelectric domain engineering in $\mathrm{BiFeO}_{3}$ thin films by second harmonic generation. Adv. Mater. 27, 4871-4876 (2015)

38. Lofland, S. E. et al. Epitaxy, texturing, and second-harmonic generation in $\mathrm{BiFeO}_{3}$ thin films. Phys. Rev. B 73, 092408 (2006).

39. Liu, S. W. et al. Domain microstructures and ferroelectric phase transition in $\mathrm{Pb}_{0.35} \mathrm{Sr}_{0.65} \mathrm{TiO}_{3}$ films studied by second harmonic generation in reflection geometry. J. Appl. Phys. 101, 104118 (2007). 
40. Jones, J., Zhu, L., Tolk, N. \& Mu, R. Investigation of ferroelectric properties and structural relaxation dynamics of polyvinylidene fluoride thin film via second harmonic generation. Appl. Phys. Lett. 103, 072901 (2013).

41. Barad, Y. et al. Domain rearrangement in ferroelectric $\mathrm{Bi}_{4} \mathrm{Ti}_{3} \mathrm{O}_{12}$ thin films studied by in situ optical second harmonic generation. J. Appl. Phys. 90, 3497-3503 (2001).

42. Mishina, E. D. et al. Domain orientation in ultrathin ( $\mathrm{Ba}, \mathrm{Sr}) \mathrm{TiO}_{3}$ films measured by optical second harmonic generation. J. Appl. Phys. 93, 6216-6222 (2003).

43. Uesu, Y., Kurimura, S. \& Yamamoto, Y. Optical second harmonic images of 90 domain structure in $\mathrm{BaTiO}_{3}$ and periodically inverted antiparallel domains in $\mathrm{LiTaO}_{3}$. Appl. Phys. Lett. 66, 2165-2167 (1995).

44. Uesu, Y., Mohri, H., Shindo, Y. \& Kurimura, S. SHG interference microscope as a tool of nondestructive observation of ferroelectric 180 domain structures. Ferroelectrics 253, 115-124 (2001).

45. Kaneshiro, J. \& Uesu, Y. Domain structure analysis of $\mathrm{Pb}\left(\mathrm{Zn}_{1 / 3} \mathrm{Nb}_{2 / 3}\right) \mathrm{O}_{3}-9 \% \mathrm{PbTiO}_{3}$ single crystals using optical second harmonic generation microscopy. Phys. Rev. $B$ 82, 184116 (2010).

46. Yokota, H., Haumont, R., Kiat, J. M., Matsuura, H. \& Uesu, Y. Second harmonic generation microscopic observations of a multiferroic $\mathrm{BiFeO}_{3}$ single crystal. Appl. Phys. Lett. 95, 082904 (2009).

47. Matsuura, H., Yokota, H., Haumont, R., Kiat, J. M. \& Uesu, Y. SHG Microscope Observations of Domain Structures of Multiferroic $\mathrm{BiFeO}_{3}$ Single Crystal. Ferroelectrics 410, 59-62 (2010).

48. Liu, W., Wang, K., Liu, Z., Shen, G. \& Lu, P. Laterally emitted surface second harmonic generation in a single ZnTe nanowire. Nano. Lett. 13, 4224-4229 (2013).

49. $\mathrm{Hu}, \mathrm{H}$. et al. Precise determination of the crystallographic orientations in single ZnS nanowires by second-harmonic generation microscopy. Nano. Lett. 15, 3351-3357 (2015).

50. McGilp, J. F. A review of optical second-harmonic and sum-frequency generation at surfaces and interfaces. J. Phys. D. Appl. Phys. 29, 1812-1821 (1996).

51. Flueraru, C., Schrader, S., Zauls, V., Dietzel, B. \& Motschmann, H. Determination of molecular orientation by angular dependence of second harmonic intensity and second harmonic phase measurements. Opt. Commun. 182, 457-466 (2000).

52. Canfield, B. K. et al. Polarization effects in the linear and nonlinear optical responses of gold nanoparticle arrays. J. Opt. A. Pure Appl. Op. 7, S110-S117 (2005).
53. Honma, T. et al. Writing of crystal line patterns in glass by laser irradiation. J. NonCryst. Solids 354, 468-471 (2008).

54. Aktsipetrov, O. A. et al. Optical second harmonic generation studies of thin ferroelectric ceramic films. Ferroelectrics 190, 143-148 (1997).

55. Woltersdorf, G., Seifert, G., Graener, H., Pignolet, A. \& Hesse, D. Nonlinear optical probing of nanocrystalline orientation in epitaxial ferroelectric thin films. J. Appl. Phys. 91, 362-366 (2002).

56. Sipe, J. E., Moss, D. J. \& Van Driel, H. M. Phenomenological theory of optical second-and third-harmonic generation from cubic centrosymmetric crystals. Phys. Rev. B 35, 1129 (1987).

57. Zavaliche, F. et al. Multiferroic $\mathrm{BiFeO}_{3}$ films: domain structure and polarization dynamics. Phase Transit. 79, 991-1017 (2006).

58. Streiffer, S. K. et al. Domain patterns in epitaxial rhombohedral ferroelectric films. I. Geometry and experiments. J. Appl. Phys. 83, 2742-2753 (1998).

59. Nelson, C. T. et al. Spontaneous vortex nanodomain arrays at ferroelectric heterointerfaces. Nano. Lett. 11, 828-834 (2011).

60. Yang, S. Y. et al. Above-bandgap voltages from ferroelectric photovoltaic devices. Nat. Nanotechnol. 5, 143-147 (2010).

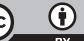

Open Access This article is licensed under a Creative Commons Attribution 4.0 International License, which permits use, sharing, adaptation, distribution and reproduction in any medium or format, as long as you give appropriate credit to the original author(s) and the source, provide a link to the Creative Commons license, and indicate if changes were made. The images or other third party material in this article are included in the article's Creative Commons license, unless indicated otherwise in a credit line to the material. If material is not included in the article's Creative Commons license and your intended use is not permitted by statutory regulation or exceeds the permitted use, you will need to obtain permission directly from the copyright holder. To view a copy of this license, visit http://creativecommons. org/licenses/by/4.0/.

C The Author(s) 2018 